\title{
Glutathione S-transferase Polymorphisms in Head and Neck Squamous Cell Carcinoma Treated with Chemotherapy and/or Radiotherapy
}

\author{
Mauricio Pereira Maniglia ${ }^{1}$, Anelise Russo ${ }^{1}$, Patrícia Matos Biselli-Chicote ${ }^{1}$, \\ Juliana Garcia de Oliveira-Cucolo ${ }^{1}$, Gabriela Helena Rodrigues-Fleming ${ }^{1}$, José \\ Victor Maniglia $^{2}$, Érika Cristina Pavarino ${ }^{1}$, Eny Maria Goloni-Bertollo ${ }^{1 *}$
}

\begin{abstract}
Background/Aim: The Glutathione S-transferases (GSTs) are important carcinogen-metabolizing enzymes. Polymorphisms involved in these enzymes can modulate the development and treatment of head and neck cancer. To investigate the association of GSTs polymorphisms with head and neck cancer and risk factors, clinical-pathological features, and survival time of the patients treated with chemotherapy and/or radiotherapy. Methods: The GST gene polymorphisms were evaluated in 197 cases and 514 controls by PCR-RFLP-Polymerase Chain Reaction Restriction Fragment Length Polymorphism. Results: The GSTP-313 was associated with a decreased risk for HNSCC ( $\mathrm{p}=0.050)$. The GSTP1 haplotype analysis revealed a higher frequency of the AC and AT haplotypes in the case group than in the control group ( $\mathrm{p}=0.013$ and $\mathrm{p}=0.019$, respectively), and the opposite for $\mathrm{G}-\mathrm{C}$ haplotype $(\mathrm{p}=0.015)$. Yet, the different combinations between the genotypes were associated with an increased risk of cancer. The study showed no association between the polymorphisms and primary tumor site, clinical-pathological characteristics, treatment (chemotherapy and/or radiotherapy) and survival time of the patients. Conclusion: The GST polymorphisms combination showed an increased risk for carcinogenesis, and studies with larger casuistry can contribute to the clarification of the role in individual patient differences for the response to chemotherapy and/or radiotherapy and identify biomarkers of susceptibility.
\end{abstract}

Keywords: Chemotherapy agents- genetic polymorphism- glutathione transferase- head and neck neoplasms

Asian Pac J Cancer Prev, 21 (6), 1637-1644

\section{Introduction}

Head and neck cancer presents an estimated 834,860 new cases in the world for 2018/2019 and comprises a heterogeneous group of tumors that originate in the squamous cells of the upper digestive tract lining epithelium, including lip, oral cavity, nasal cavity, paranasal sinus, pharynx, larynx (IARC, 2019). It is a multifactorial disease, influenced by age, gender, environmental factors such as smoking habits, alcohol consumption, HPV infection, genetic factors such as gene polymorphisms and exposure to radiation solar UVA (lips cancer) (Mirghani et al., 2017).

Regarding genetic factors, studies show that polymorphisms in genes that encode drug metabolism enzymes used in chemotherapy can cause variability in drug responses and modulate treatment outcomes in head and neck squamous cell carcinoma (HNSCC) cases, thus individualized treatment can improve the better patient response (Ruwali et al., 2009; Galbiatti et al., 2013; Carron et al., 2017; Hasegawa et al., 2018; Macedo et al., 2019).

Drug metabolism or xenobiotic metabolism involves two major types of enzymes. Conjugated enzymes/ Phase II involves conjugation with an endogenous substrate (glutathione, sulfate, glucose, acetate) by glutathione-S-transferase (GSTs) enzymes. GSTs act as inactivating enzymes for Phase I products/ mediated oxidative metabolism by cytochrome $\mathrm{P} 450$ and microsomal epoxide hydrolase, leading to hydrophilic metabolite capable of being excreted for homeostatic control and detoxification of lipophilic xenobiotics which occurs in the Phase III (Marchewka et al., 2017).

Genetic variations in glutathione S-transferases have been associated with changes in the catalytic activities of the enzyme and can be associated with increased DNA adducts and increasing the risk of cancer (Chatterjee and Gupta, 2018; Singh and Ghosh, 2019; Zhang et al., 2019). Also, low response to chemotherapy applying cisplatin 
for oral cancer was found in cases of GSTP1 AA313 wild genotype, demonstrating the possible role of these polymorphisms in patient responses to chemotherapy (Ruwali et al., 2009).

Thus, studies concerning these polymorphisms are important to identify biomarkers of susceptibility to HNSCC. Considering the evidence presented, this study aimed to investigate the association of GSTM1 and GSTT1 null genotypes and single nucleotide polymorphisms (SNPS) A313G and C341T of the GSTP1 gene with head and neck cancer, as well as to verify the association between these polymorphisms and the anatomical site of tumor occurrence, clinical-pathological characteristics, relapse-free time and survival of patients with head and neck cancer treated with chemotherapy and/or radiotherapy.

\section{Materials and Methods}

\section{Patients and Methods \\ Sample characterization}

A total of 711 individuals were included in the present retrospective case-control study, 197 patients composing the case group, diagnosed with HNSCC during follow-up at the Otorhinolaryngology and Head and Neck Surgery Service belonging to the FAMERP/FUNFARME hospital medical complex, in São José do Rio Preto - SP, and 514 healthy individuals with no history of neoplasia obtained at the São José do Rio Preto Base Hospital blood center, comprising the control group. Patient and control data were collected through standardized questionnaires. All samples were obtained after the signing of an Informed Consent Term. The study was approved by the Research Ethics Committee (No. 697,896).

The inclusion criterion for the case group was pathological biopsy confirmation of primary tumors diagnosed as HNSCC treated with chemotherapy and/or radiotherapy at the reference hospital in Southwest São Paulo by FAMERP/FUNFARME Otorhinolaryngology, Head and Neck Surgery of Services. Exclusion criteria for the case group were patients with head and neck carcinomas other than squamous cell carcinoma and patients who were not submitted to chemotherapy and/ or radiotherapy.

Regarding the control group, the inclusion criteria were healthy individuals aged $\geq 40$ years regardless of gender and without cancer or any family history of neoplasia. Exclusion criteria for the control group were individuals with health-related complaints, changes in blood counts, infectious-contagious diseases, patients presenting chronic diseases, a family history of cancer and $<40$ years of age.

Subjects who had smoked $>100$ cigarettes during their lifetime were considered smokers, and subjects who consumed more than four alcohol doses per week (one dose corresponding to liquor $-30 \mathrm{~mL}$, glass of wine $-102 \mathrm{~mL}$ containing $12 \%$ of alcohol, or can of beer $-340 \mathrm{~mL}$ ) were considered alcohol consumers. The analyzed variables were gender, age, smoking habit, alcohol consumption, primary site and clinical-pathological features of the tumor. Patient treatment included radiotherapy and/or chemotherapy and the main chemotherapeutic agents were cisplatin, methotrexate, and 5-fluorouracil.

\section{Clinical characterization}

The neoplasias were categorized concerning the primary site, namely oral cavity, pharynx, and larynx. American Joint Committee for Cancer - AJCC (Edge and Compton, 2010) and Union for International Cancer Control - UICC (Sobin, 2011) criteria for tumor classification involve tumor extension (T), nodal metastasis $(\mathrm{N})$ and distant metastasis $(\mathrm{M})$. T classification was categorized as smaller (T1, T2) and larger (T3, T4) tumors. $\mathrm{N}$ classification was defined as the absence (N0) and presence (N1, N2, N3) of nodal metastasis. $\mathrm{M}$ classification was dichotomized into absence (M0) or presence (M1) of distant metastasis. The stages were divided into early (stages I and II) and advanced disease (III and IV) (Lee, 2011; Sobin, 2011). Diagnosis, primary anatomical site, TNM classification, and certain clinical information were obtained from patient medical records, included in the study and evaluated by the study author (an otorhinolaryngologist and head and neck surgeon).

\section{Molecular analysis}

Genomic DNA was extracted from total peripheral blood, according to Salazar (Salazar et al., 1998). GSTT1 and GSTM1 polymorphisms: performed by Multiplex Polymerase Chain Reaction (PCR), using the CYP1Al gene as an internal positive control for primer amplification (Abdel-Rahman et al., 1996). PCR products were analyzed on $1.5 \%$ agarose gels stained with ethidium bromide.

A313G polymorphism of the GSTP1 gene: The PCR products, 176 base pair (bp) were treated with the Bsmal enzyme for application of the PCR-RFLP technique: AA wild genotype (176bp); GG polymorphic genotype (91 and $85 \mathrm{bp}$ ) and AG heterozygous (176, 91 and $85 \mathrm{bp}$ ) (Harries et al., 1997). C341T polymorphism of GSTP1 gene: The PCR products, 420 base pair (bp) were treated with Acil for application the PCR-RFLP technique: CC wild genotype (247, 118 and $55 \mathrm{bp}$ ); CT heterozygous genotype (365, 247, 118 and $55 \mathrm{bp}$ ) and TT polymorphic genotype (365 and 55 bp) (Watson et al., 1998). The enzymatic digestion products were then analyzed after electrophoresis on $2.5 \%$ agarose gels stained with ethidium bromide. The molecular evaluation was repeated in $10 \%$ of the samples to confirm the genotyping of the investigated polymorphisms.

\section{Statistical analysis}

Genotypic frequencies of the A313G (rs1695) and C341T (rs749174) GSTP1 polymorphisms were evaluated for the Hardy-Weinberg Equilibrium (HWE) by the Chi-Square Test $\left(\chi^{2}\right)$ using the BioEstat program, version 5.0. The haplotype analysis of the A313G and C341T GSTP1polymorphisms was performed using the Haploview program, version 4.2. The SNPStats program (available at http://bioinfo.iconcologia.net/SNPstats_web) was used to perform the multiple logistic regression to evaluate the effect of the variables analyzed and cancer association between the genetic models of the polymorphisms and the development of HNSCC, adjusted for age, gender, and 
smoking and drinking habits. The analysis included age (reference: $<$ median of the case group), gender (reference: female), smoking habit (reference: non-smokers) and alcohol consumption (reference: non-consumers). The effect of the polymorphisms was evaluated in the models as (1) codominant (heterozygous vs wild-type homozygous and polymorphic homozygous vs wild-type homozygous); (2) dominant (heterozygous + polymorphic homozygous vs wild-type homozygous); (3) recessive (homozygous polymorphic vs wild- type homozygous + heterozygous); (4) overdominant (heterozygous vs wild-type homozygous + polymorphic homozygous); or (5) additive (polymorphic homozygous with $2+$ heterozygous vs wild-type homozygous). The Instat Program was used to evaluate the association of the combined GSTT1, GSTM1, GSTP1 A313G* and GSTP1 C341T* polymorphisms in HNSCC risk.

Concerning size $(\mathrm{T})$, tumors were classified as $\mathrm{T} 1$ and T2 (small) and T3 and T4 (large). According to regional lymph node involvement $(\mathrm{N})$, cases were classified as negative (N0) or positive (N1, N2, and N3). Tumors were classified as M0 (absence of distant metastases) and M1 (with metastases) regarding metastasis. Polymorphism effects on relapse and survival time of patients presenting HNSCC were analyzed using the Kaplan Meier Curve and Log Rank test. The results were presented as the Odds Ratio (OR) and 95\% confidence interval (CI - 95\%). The level of significance was established as $5 \%(\mathrm{p} \leq 0.05)$.

\section{Results}

A total of 197 patients and 514 controls were analyzed. The median age was 59 years old, ranging from 34 to 83 in the case group and 44 years old, ranging from 24 to 90 in the control group. Males were predominant in both case $(90.0 \%)$ and control $(71.8 \%)$ groups. The Multiple Logistic Regression analysis for socio-demographic characteristics and risk factors showed that in the Case group male gender $(\mathrm{OR}=2.13 ; 95 \% \mathrm{CI}=1.03-4.38$; $\mathrm{p}=0.040)$, age $\geq 59$ years $(\mathrm{OR}=11.66 ; 95 \% \mathrm{CI}=$ $6.51-20.86 ; \mathrm{p}<0.001)$, smoking habit $(\mathrm{OR}=11.00 ; 95 \%$ $\mathrm{CI}=5.71-21.20 ; \mathrm{p}<0.001)$ and alcoholic habit $(\mathrm{OR}=$ 4.14; 95\% CI $=2.25-7.64 ; \mathrm{p}<0.001$ ) were associated with greater susceptibility to the development of this neoplasm (Table 1).

In the Case group, the genotype frequencies of GSTP1-A313G were $56.7 \%$ for the A/A, $34.8 \%$ for $\mathrm{A} / \mathrm{G}$ and $8.4 \%$ for $\mathrm{G} / \mathrm{G}$, while in the control group, these frequencies were $44.2 \%, 43.0 \%$, and $12.8 \%$, respectively. As showed in the Table 1, the GSTP1-313 A/G + G/G genotypes was associated in the dominant model with a decreased risk for $\mathrm{HNSCC}(\mathrm{OR}=0.64 ; 95 \% \mathrm{CI}=0.42$ $-1.00 ; \mathrm{p}=0.050)$. In contrast, for GSTM1 and GSTT1 null genotype and SNP GSTP1-C341T, no significant differences were found in the evaluated two groups.

We evaluated the combined effect of the four polymorphisms studied (Table 2), an increase risk of HNSCC was observed in the presence of the duple combination: GSTT1 non-null/GSTM1 null genotypes $(\mathrm{OR}=1.93 ; 95 \% \mathrm{CI}: 1.15-3.24 ; \mathrm{p}=0.012)$, GSTP1-313AA/-341CT+TT $(\mathrm{OR}=6.12$; 95\%CI: 1.87 -
Table 1. Sociodemographic Characteristics, Risk Factors, and Polymorphisms, GSTP1 A313G, GSTP1 C341T, GSTT1 and GSTM1 in Patients with Head and Neck Cancer and Controls.

\begin{tabular}{|c|c|c|c|c|}
\hline Variables & $\begin{array}{c}\text { Case } \\
\mathrm{n}=197(\%)\end{array}$ & $\begin{array}{c}\text { Control } \\
\mathrm{n}=514(\%)\end{array}$ & OR $(95 \% \mathrm{CI})$ & $\begin{array}{c}P \text { - } \\
\text { value }\end{array}$ \\
\hline \multicolumn{5}{|l|}{ Gender } \\
\hline Female & $19(10.0)$ & $145(28.0)$ & 1 & 0.040 \\
\hline Male & $178(90.0)$ & $369(72.0)$ & $2.13(1.03-4.38)$ & \\
\hline \multicolumn{5}{|l|}{ Age (Median) } \\
\hline$<59$ & $97(49.0)$ & $448(87.0)$ & 1 & \\
\hline$\geq 59$ & $100(51.0)$ & $66(13.0)$ & $11.66(6.51-20.86)$ & $<0.001$ \\
\hline \multicolumn{5}{|l|}{ Smoking Habit } \\
\hline Non-smoke & $20(10.0)$ & $309(60.0)$ & 1 & $<0.001$ \\
\hline Smoke & $177(90.0)$ & $205(40.0)$ & $11.00(5.71-21$ & 20) \\
\hline \multicolumn{5}{|l|}{ Alcoholic Habit } \\
\hline Non-alcoholic & $28(14.0)$ & $260(51.0)$ & 1 & $<0.001$ \\
\hline Alcoholic & $169(86.0)$ & $254(49.0)$ & $4.14(2.25-7.64)$ & \\
\hline \multicolumn{5}{|l|}{ Polymorphisms } \\
\hline \multicolumn{5}{|l|}{ GSTT1 } \\
\hline$+/+$ & $140(71.1)$ & $402(78.2)$ & 1 & 0.610 \\
\hline $0 / 0$ & 57 (28.9) & $112(21.8)$ & $1.14(0.69-1.87)$ & \\
\hline \multicolumn{5}{|l|}{ GSTMI } \\
\hline$+/+$ & $98(49.8)$ & $275(53.5)$ & 1 & 0.400 \\
\hline $0 / 0$ & $99(50.2)$ & $239(6.5)$ & $1.20(0.78-1.85)$ & \\
\hline \multicolumn{5}{|l|}{$G S T P 1-A 313 G^{a}$} \\
\hline \multicolumn{5}{|l|}{ Codominant } \\
\hline $\mathrm{A} / \mathrm{A}$ & $101(56.7)$ & $227(44.2)$ & 1 & 0.140 \\
\hline $\mathrm{A} / \mathrm{G}$ & $62(34.8)$ & $221(43)$ & $0.66(0.41-1.05)$ & \\
\hline $\mathrm{G} / \mathrm{G}$ & $15(8.4)$ & $66(12.8)$ & $0.61(0.29-1.25)$ & \\
\hline \multicolumn{5}{|l|}{ Dominant } \\
\hline $\mathrm{A} / \mathrm{A}$ & $101(56.7)$ & $227(44.2)$ & 1 & 0.050 \\
\hline A/G-G/G & $77(43.3)$ & $287(55.8)$ & $0.64(0.42-1.00)$ & \\
\hline \multicolumn{5}{|l|}{ Recessive } \\
\hline $\mathrm{A} / \mathrm{A}-\mathrm{A} / \mathrm{G}$ & $163(91.6)$ & $448(87.2)$ & 1 & 0.360 \\
\hline $\mathrm{G} / \mathrm{G}$ & $15(8.4)$ & $66(12.8)$ & $0.73(0.36-1.46)$ & \\
\hline \multicolumn{5}{|l|}{ Overdominant } \\
\hline A/A-G/G & $116(65.2)$ & $293(57.0)$ & 1 & 0.16 \\
\hline $\mathrm{A} / \mathrm{G}$ & $62(34.8)$ & $221(43.0)$ & $0.72(0.46-1.14)$ & \\
\hline Additive & --- & --- & $0.74(0.53-1.02)$ & 0.062 \\
\hline \multicolumn{5}{|l|}{ GSTP1-C341T } \\
\hline \multicolumn{5}{|l|}{ Codominant } \\
\hline $\mathrm{C} / \mathrm{C}$ & 173 (89.6) & $460(89.5)$ & 1 & 0.260 \\
\hline $\mathrm{C} / \mathrm{T}$ & $20(10.4)$ & $50(9.7)$ & $0.80(0.40-1-60)$ & \\
\hline $\mathrm{T} / \mathrm{T}$ & $0(0)$ & $4(0.8)$ & 0 & \\
\hline \multicolumn{5}{|l|}{ Dominant } \\
\hline $\mathrm{C} / \mathrm{C}$ & 173 (89.6) & $460(89.5)$ & 1 & 0.380 \\
\hline $\mathrm{C} / \mathrm{T}-\mathrm{T} / \mathrm{T}$ & $20(10.4)$ & $54(10.5)$ & $0.74(0.37-1.46)$ & \\
\hline \multicolumn{5}{|l|}{ Recessive } \\
\hline $\mathrm{C} / \mathrm{C}-\mathrm{C} / \mathrm{T}$ & $193(100)$ & $510(99.2)$ & 1 & 0.150 \\
\hline $\mathrm{T} / \mathrm{T}$ & $0(0)$ & $4(0.8)$ & 0 & \\
\hline \multicolumn{5}{|l|}{ Overdominant } \\
\hline $\mathrm{C} / \mathrm{C}-\mathrm{T} / \mathrm{T}$ & 173 (89.6) & $464(90.3)$ & 1 & 0.550 \\
\hline $\mathrm{C} / \mathrm{T}$ & $20(10.4)$ & $50(9.7)$ & $0.81,(0.41-1.62)$ & \\
\hline Additive & --- & --- & $0.70(0.37-1.33)$ & 0.270 \\
\hline
\end{tabular}

a, Odds Ratio (OR) adjusted for age, gender, and alcohol and smoking habits and polymorphisms significant $\mathrm{p}$ values for $\mathrm{p}<0.05$ a Amplification was possible for 692 individuals of the case group

${ }^{\mathrm{b}}$ Amplification was possible for 707 individuals of the case groupd. 
Table 2. Association of the Combined GSTT1, GSTM1, GSTP1 A313G* and GSTP1 C341T* Genotypes in Patients with Head and Neck Cancer and Controls, Adjusted for Gender, Age, Smoking and Alcohol Consumption

\begin{tabular}{|c|c|c|c|c|c|c|c|}
\hline GSTT1 & GSTM1 & & & Case (187) & Control (514) & OR $(95 \% \mathrm{IC})$ & $P$ value \\
\hline Positve & Positive & & & 66 & 220 & 1 & \\
\hline Positive & Negative & & & 74 & 182 & $1.355(0.92-1.99)$ & 0.140 \\
\hline Negative & Positive & & & 32 & 55 & $1.939(1.158-3.247)$ & 0.012 \\
\hline Negative & Negative & & & 25 & 57 & $1.462(0.847-2.521)$ & 0.191 \\
\hline GSTP1 A313G* & GSTP1 C341T* & & & Case (178) & Control (514) & OR (95\%IC) & $P$ value \\
\hline AA & $\mathrm{CC}$ & & & 91 & 223 & 1 & \\
\hline AA & $\mathrm{CT} / \mathrm{TT}$ & & & 10 & 4 & $6.12(1.87-20.04)$ & 0.001 \\
\hline $\mathrm{AG} / \mathrm{GG}$ & $\mathrm{CC}$ & & & 67 & 237 & $0.69(0.48-0.99)$ & 0.057 \\
\hline $\mathrm{AG} / \mathrm{GG}$ & $\mathrm{CT} / \mathrm{TT}$ & & & 10 & 50 & $0.49(0.23-1.00)$ & 0.056 \\
\hline GSTT1 & GSTM1 & GSTP1 A313G* & & Case (178) & Control (514) & OR $(95 \% \mathrm{IC})$ & $P$ value \\
\hline Positve & Positive & $\mathrm{AA}$ & & 29 & 96 & 1 & \\
\hline Positive & Positive & AG/GG & & 28 & 124 & $0.97(0.54-1.73)$ & 1 \\
\hline Negative & Positive & AA & & 17 & 22 & $3.33(1.57-7.05)$ & 0.002 \\
\hline Negative & Positive & AG/GG & & 13 & 33 & $1.69(0.79-3.62)$ & 0.214 \\
\hline Positive & Negative & $\mathrm{AA}$ & & 42 & 86 & $2.10(1.21-3.63)$ & 0.008 \\
\hline Positive & Negative & AG/GG & & 27 & 96 & $1.21(0.67-2.18)$ & 0.549 \\
\hline Negative & Negative & $\mathrm{AA}$ & & 13 & 23 & $2.43(1.10-5.37)$ & 0.042 \\
\hline Negative & Negative & $\mathrm{AG} / \mathrm{GG}$ & & 9 & 34 & $1.14(0.49-2.63)$ & 0.827 \\
\hline GSTT1 & GSTM1 & GSTP1 C341T & & Case (193) & Control (514) & OR $(95 \% \mathrm{IC})$ & $P$ value \\
\hline Positve & Positive & $\mathrm{CC}$ & & 57 & 198 & 1 & \\
\hline Positive & Positive & $\mathrm{CT} / \mathrm{TT}$ & & 8 & 22 & $1.26(0.53-2.98)$ & 0.645 \\
\hline Negative & Positive & $\mathrm{CC}$ & & 28 & 49 & $1.98(1.14-3.44)$ & 0.017 \\
\hline Negative & Positive & $\mathrm{CT} / \mathrm{TT}$ & & 2 & 6 & $1.15(0.22-5.89)$ & 1 \\
\hline Positive & Negative & $\mathrm{CC}$ & & 66 & 163 & $1.40(0.93-2.12)$ & 0.116 \\
\hline Positive & Negative & $\mathrm{CT} / \mathrm{TT}$ & & 8 & 19 & $1.46(0.60-3.51)$ & 0.470 \\
\hline Negative & Negative & $\mathrm{CC}$ & & 22 & 50 & $1.52(0.85-2.73)$ & 0.162 \\
\hline Negative & Negative & $\mathrm{CT} / \mathrm{TT}$ & & 2 & 7 & $0.99(0.20-4.91)$ & 1 \\
\hline GSTT1 & GSTM1 & GSTP1 A313G* & GSTP1 C341T & Case (178) & Control (514) & OR $(95 \% \mathrm{IC})$ & $P$ value \\
\hline Positve & Positive & $\mathrm{AA}$ & $\mathrm{CC}$ & 25 & 94 & 1 & \\
\hline Positive & Positive & AA & $\mathrm{CT} / \mathrm{TT}$ & 4 & 2 & $7.52(1.30-43.45)$ & 0.025 \\
\hline Positive & Positive & $\mathrm{AG} / \mathrm{GG}$ & $\mathrm{CC}$ & 24 & 104 & $0.86(0.46-1.62)$ & 0.749 \\
\hline Positive & Positive & $\mathrm{AG} / \mathrm{GG}$ & $\mathrm{CT} / \mathrm{TT}$ & 4 & 20 & $0.75(0.23-2.40)$ & 0.784 \\
\hline Negative & Positive & AA & $\mathrm{CC}$ & 16 & 21 & $2.86(1.30-6.28)$ & 0.01 \\
\hline Negative & Positive & AA & $\mathrm{CT} / \mathrm{TT}$ & 1 & 1 & $3.76(0.22-62.28)$ & 0.385 \\
\hline Negative & Positive & $\mathrm{AG} / \mathrm{GG}$ & $\mathrm{CC}$ & 12 & 28 & $1.61(0.71-3.61)$ & 0.281 \\
\hline Negative & Positive & $\mathrm{AG} / \mathrm{GG}$ & $\mathrm{CT} / \mathrm{TT}$ & 1 & 5 & $0.75(0.08-6.73)$ & 1 \\
\hline Positive & Negative & AA & $\mathrm{CC}$ & 38 & 85 & $1.68(0.93-3.01)$ & 0.106 \\
\hline Positive & Negative & AA & $\mathrm{CT} / \mathrm{TT}$ & 4 & 1 & $15.04(1.60-140.68)$ & 0.01 \\
\hline Positive & Negative & $\mathrm{AG} / \mathrm{GG}$ & $\mathrm{CC}$ & 23 & 78 & $1.10(0.58-2.10)$ & 0.870 \\
\hline Positive & Negative & $\mathrm{AG} / \mathrm{GG}$ & $\mathrm{CT} / \mathrm{TT}$ & 4 & 18 & $0.83(0.25-2.69)$ & 1 \\
\hline Negative & Negative & AA & $\mathrm{CC}$ & 12 & 23 & $1.96(0.85-4.48)$ & 0.118 \\
\hline Negative & Negative & $\mathrm{AA}$ & $\mathrm{CT} / \mathrm{TT}$ & 1 & 0 & $11.11(0.43-281.37)$ & 0.216 \\
\hline Negative & Negative & $\mathrm{AG} / \mathrm{GG}$ & $\mathrm{CC}$ & 8 & 27 & $1.11(0.45-2.75)$ & 0.817 \\
\hline Negative & Negative & $\mathrm{AG} / \mathrm{GG}$ & $\mathrm{CT} / \mathrm{TT}$ & 1 & 7 & $0.53(0.06-4.57)$ & 1 \\
\hline
\end{tabular}

Odds Ratio (OR) adjusted for age, gender, smoking habit and alcohol consumption

20.04, $\mathrm{p}=0.001)$; triple combination: GSTT1 non-null/ GSTM1 null/GSTP1-313AA (OR $=3.33 ; 95 \% \mathrm{CI}$ : 1.57-7.05, $\mathrm{p}=0.002)$, GSTT1 null/GSTM1 non- null/ GSTP1-313AA $(\mathrm{OR}=2.10$; 95\%CI: 1.21-3.63, $\mathrm{p}=0.008)$, GSTT1 non-null/GSTM1non- null/GSTP1-313AA $(\mathrm{OR}=2.43 ; 95 \% \mathrm{CI}: 1.10-5.37, \mathrm{p}=0.042)$, GSTT1
non-null/GSTM1 null/GSTP1-341CC (OR = 1.98; 95\%CI: 1.14-3.44, $\mathrm{p}=0.017)$, and fourth combination: GSTT1 null/GSTM1 null/GSTP1-313AA/GSTP1-341CT+TT $(\mathrm{OR}=7.52$; 95\%CI: 1.30-43.45, $\mathrm{p}=0.025)$, GSTT1 null/ GSTM1 non-null/GSTP1-313AA/GSTP1-341CT+TT(OR $=15.04 ; 95 \% \mathrm{CI}: 1.60-140.68, \mathrm{p}=0.010)$ and GSTT1

1640 Asian Pacific Journal of Cancer Prevention, Vol 21 
Table 3. Haplotype Frequency Distribution of GSTP1 Polymorphisms Gene between Case and Control Groups

\begin{tabular}{lccc}
\hline & A313G / C341T GSTP1 polymorphisms \\
Haplotype & HNSCC (\%) & Control (\%) & $P$-value \\
\hline A-C & 72.0 & 65.0 & 0.013 \\
G-C & 23.0 & 30.0 & 0.015 \\
G-T & 3.0 & 5.0 & 0.085 \\
A-T & 3.0 & 0.9 & 0.019 \\
\hline
\end{tabular}

non-null/GSTM1 null/GSTP1-313AA/GSTP1-341CC

$(\mathrm{OR}=2.86 ; 95 \% \mathrm{CI}: 1.30-6.28, \mathrm{p}=0.010)$.
Genotypic frequencies for the A313G and C341T GSTP1 polymorphisms were in Hardy-Weinberg equilibrium in both groups $(\mathrm{p}>0.05)$. The A313G/C341T GSTP1 haplotypes (Table 3) revealed a higher frequency of the two wild alleles (A-C) in the case group than in the control group (72.0 and 65.0, respectively; $p=0.013$ ). Similarly, a higher frequency of the A-T haplotype was also found in the case group than in the control group (3.0 and 0.9 , respectively; $\mathrm{p}=0.019$ ). However, the opposite was observed for the G-C haplotype, which was more

Table 4. GSTT1, GSTM1, A313G GSTP1 and C341T GSTP1 in Relation to the Primary Sites of Head and Neck Cancer Tumors

\begin{tabular}{|c|c|c|c|c|c|c|c|c|c|}
\hline & \multicolumn{3}{|c|}{$\begin{array}{c}\text { Oral cavity } \\
\mathrm{n}=87(44.0 \%)\end{array}$} & \multicolumn{3}{|c|}{$\begin{array}{c}\text { Larynx } \\
\mathrm{n}=64(32.0 \%)\end{array}$} & \multicolumn{3}{|c|}{$\begin{array}{c}\text { Pharynx } \\
\mathrm{n}=37(19.0 \%)\end{array}$} \\
\hline & n $(\%)$ & OR $(95 \% \mathrm{CI})$ & $\mathrm{p}$ & $\mathrm{n}(\%)$ & OR $(95 \% \mathrm{CI})$ & $\mathrm{p}$ & $\mathrm{n}(\%)$ & OR $(95 \% \mathrm{CI})$ & $\mathrm{p}$ \\
\hline \multicolumn{10}{|l|}{ GSTM1 } \\
\hline Positive & $43(49.0)$ & Reference & & $33(51.0)$ & Reference & & $19(51.0)$ & Reference & \\
\hline Negative & $44(51.0)$ & $0.98(0.53-1.83)$ & 0.955 & $31(49.0)$ & $1.02(0.51-2.04)$ & 0.945 & $18(49.0)$ & $0.78(0.36-1.73)$ & 0.545 \\
\hline \multicolumn{10}{|l|}{ GSTT1 } \\
\hline Positive & $63(72.0)$ & Reference & & $48(75.0)$ & Reference & & $27(73.0)$ & Reference & \\
\hline Negative & $24(28.0)$ & $0.83(0.41-1.67)$ & 0.596 & $16(25.0)$ & $0.77(0.35-1.70)$ & 0.513 & $10(27.0)$ & $0.97(0.40-2.38)$ & 0.947 \\
\hline \multicolumn{10}{|c|}{$\mathrm{A} 313 \mathrm{G} \mathrm{GSTP} 1^{a}$} \\
\hline AA & $46(57.5)$ & Reference & & $28(50.0)$ & Reference & & $22(59.0)$ & Reference & \\
\hline $\mathrm{AG} / \mathrm{GG}$ & $34(42.5)$ & 0.89 (0.48-1.67) & 0.727 & $28(50.0)$ & $1.47(0.74-2.93)$ & 0.269 & $11(41.0)$ & $0.66(0.29-1.48)$ & 0.312 \\
\hline \multicolumn{10}{|c|}{ C341T GSTP $1^{b}$} \\
\hline $\mathrm{CC}$ & $76(90.5)$ & Reference & & $57(90.5)$ & Reference & & 33 (91.6) & Reference & \\
\hline $\mathrm{CT}$ & $09(9.5)$ & $1.07(0.39-2.92)$ & 0.899 & $06(9.5)$ & $0.48(0.14-1.65)$ & 0.243 & $03(8.4)$ & $1.44(0.43-4.82)$ & 0.554 \\
\hline
\end{tabular}

Odds Ratio (OR) adjusted for age, gender, smoking habit and alcohol consumption. ${ }^{a}$ Amplification was possible for 80,56 and 33 patients for oral cavity, larynx and pharynx, respectively. ${ }^{\mathrm{b} A m p l i f i c a t i o n}$ was possible for 85,63 and 36 patients for oral cavity, larynx and pharynx, respectively.

Table 5. Clinical-Histopathological Features in Relation to GSTT1, GSTM1, A313G GSTP1 and C341T GSTP1 Polymorphisms in Patients with Head and Neck Cancer

\begin{tabular}{|c|c|c|c|c|c|c|c|c|c|c|c|c|}
\hline & \multicolumn{4}{|c|}{ Tumor extension (191) } & \multicolumn{4}{|c|}{ Regional lymph node involvement (195) } & \multirow[b]{2}{*}{$\begin{array}{l}\text { Early } \\
\text { n (\%) }\end{array}$} & \multirow[b]{2}{*}{$\begin{array}{c}\text { Advanced } \\
\mathrm{n}(\%)\end{array}$} & \multirow[b]{2}{*}{$\begin{array}{c}\text { OR+ } \\
(95 \% \mathrm{CI})\end{array}$} & \multirow[b]{2}{*}{$\mathrm{p}$} \\
\hline & $\begin{array}{l}\mathrm{T} 1 / \mathrm{T} 2 \\
\mathrm{n}(\%)\end{array}$ & $\begin{array}{l}\mathrm{T} 3 / \mathrm{T} 4 \\
\mathrm{n}(\%)\end{array}$ & $\begin{array}{c}\text { OR }+ \\
(95 \% \mathrm{CI})\end{array}$ & $\mathrm{p}$ & $\begin{array}{l}\mathrm{N}=0 \\
\mathrm{n}(\%)\end{array}$ & $\begin{array}{l}\mathrm{N} \geq 1 \\
\mathrm{n}(\%)\end{array}$ & $\begin{array}{c}\text { OR+ } \\
(95 \% \mathrm{CI})\end{array}$ & $\mathrm{p}$ & & & & \\
\hline \multicolumn{13}{|l|}{ GSTM1 } \\
\hline Positive & $40(51.3)$ & $56(49.5)$ & 1 & & $48(44.8)$ & $48(54.5)$ & 1 & & $31(50.0)$ & $64(48.5)$ & 1 & \\
\hline Negative & $38(48.7)$ & $57(50.5)$ & $\begin{array}{c}1.06 \\
(0.57-1.97)\end{array}$ & 0.860 & $59(55.2)$ & $40(45.5)$ & $\begin{array}{c}0.74 \\
(0.40-1.38)\end{array}$ & 0.350 & $31(50.0)$ & $68(51.5)$ & $\begin{array}{c}1.15 \\
(0.59- \\
2.24)\end{array}$ & 0.673 \\
\hline \multicolumn{13}{|l|}{ GSTT1 } \\
\hline Positive & $55(70.5)$ & $82(72.6)$ & 1 & & $78(72.8)$ & $61(69.3)$ & 1 & & $44(70.9)$ & $95(71.9)$ & 1 & \\
\hline Negative & $23(29.5)$ & $31(27.4)$ & $\begin{array}{c}0.82 \\
(0.41-1.65)\end{array}$ & 0.580 & $29(27.2)$ & $27(30.7)$ & $\begin{array}{c}1.23 \\
(0.62-2.48)\end{array}$ & 0.550 & $18(29.1)$ & $37(28.1)$ & $\begin{array}{c}1.22 \\
(0.57- \\
2.63)\end{array}$ & 0.605 \\
\hline \multicolumn{13}{|c|}{$\mathrm{A} 313 \mathrm{G} \mathrm{GSTP} 1^{a}$} \\
\hline AA & $42(59.0)$ & $55(54.4)$ & 1 & & $55(57.9)$ & $46(56.0)$ & 1 & & $34(60.7)$ & $67(55.8)$ & 1 & \\
\hline $\mathrm{AG} / \mathrm{GG}$ & $29(41.0)$ & $46(45.6)$ & $\begin{array}{c}1.16 \\
(0.62-2.16)\end{array}$ & 0.650 & $40(42.1)$ & $36(44.0)$ & $\begin{array}{l}1.13 \\
(0.61- \\
2.11)\end{array}$ & 0.700 & $22(39.3)$ & $53(44.2)$ & $\begin{array}{c}1.05 \\
(0.53- \\
2.06)\end{array}$ & 0.896 \\
\hline \multicolumn{13}{|c|}{ C341T GSTP $1^{b}$} \\
\hline $\mathrm{CC}$ & $71(92.0)$ & $98(89.0)$ & 1 & & $91(80.5)$ & $80(91.0)$ & 1 & & $56(91.8)$ & $114(88.4)$ & 1 & \\
\hline CT & $06(8.0)$ & $12(11.0)$ & $\begin{array}{c}0.93 \\
(0.34-2.54)\end{array}$ & 0.890 & $12(19.5)$ & $08(9.0)$ & $\begin{array}{c}0.72 \\
(0.26-2.00)\end{array}$ & 0.530 & $05(8.2)$ & 15 (11.6) & $\begin{array}{c}1.27 \\
(0.40- \\
3.98)\end{array}$ & 0.685 \\
\hline
\end{tabular}

Odds Ratio (OR) adjusted for age, gender, smoking habit and alcohol consumption. ${ }^{a}$ Amplification was possible for 172,177 and 176 patients for tumor extension, regional lymph node involvement and TNM, respectively. ${ }^{\mathrm{b} A m p l i f i c a t i o n}$ was possible for 187 , 191 and 190 patients for tumor extension, regional lymph node involvement and TNM, respectively 
Table 6. GSTT1, GSTM1, A313G GSTP1 and C341T GSTP1 Polymorphisms in Relation to Recurrence and Survival of Patients with Head and Neck Cancer

\begin{tabular}{lcccc}
\hline Polymorphisms & Relapse (12 months) & $P$-value Log Rank & Survival (12 months) & $P$-value Log Rank \\
\hline $\begin{array}{l}\text { GSTT1 } \\
\text { Positive }\end{array}$ & $23.0 \%$ & & & 0.135 \\
Negative & $30.0 \%$ & 0.467 & $85.0 \%$ & $72.0 \%$ \\
GSTM1 & & & & 0.314 \\
Positive & $26.0 \%$ & 0.717 & $85.0 \%$ & 0.487 \\
Negative & $22.0 \%$ & & $80.0 \%$ & \\
A313G GSTP1 & & & & \\
AA & $24.0 \%$ & 0.450 & $88.0 \%$ & 0.566 \\
AG/GG & $23.0 \%$ & & $83.0 \%$ & \\
C341T GSTP1 & & & $82.0 \%$ & \\
CC & $25.0 \%$ & 0.164 & & \\
CT/TT & $12.0 \%$ & & & \\
\hline
\end{tabular}

frequent in the control group than in the case group $(23.0$ and 30.0, respectively; $\mathrm{p}=0.015$ ).

Regarding the anatomical site, $44.0 \%$ of the tumors were found in the oral cavity, $32.0 \%$ in the larynx, $19.0 \%$ in the pharynx, and $5.0 \%$ of the individuals presented tumors of unknown origin. Considering tumor size, $57.0 \%$ of the patients presented large tumors (six tumors were not possible to classify in T categories). No involvement of regional lymph nodes (N0) was observed in 55\% of the cases (two tumors were not possible to classify in $\mathrm{N}$ categories). Only $3.0 \%$ of patients presented distant metastases (M1). The staging was evaluated for 194 patients. The present study demonstrated no association between the investigated polymorphisms and primary tumor site (Table 4), as well as between the genotypes and the clinical-pathological tumor characteristics (Table 5). Analyzing patient treatment, the most common was a radiotherapy and chemotherapy association with $69.0 \%$, against 26.0 and $5.0 \%$ for exclusive radiotherapy and exclusive chemotherapy, respectively.

The Kaplan-Meier Curve analysis indicated that relapse-free and survival time in patients with GSTM1, or GSTT1, or GSTP1 polymorphism presented no significant difference concerning allele carriers and non-carriers patients (Table 6).

\section{Discussion}

The present study confirmed data from the literature, that report the predominance of cases between the fourth and sixth decade of life, and that smoking and alcohol consumption is strongly related to increased risk of developing this type of cancer (Choudhury et al., 2015; Rodrigues-Fleming et al., 2018). The studies carried out in Northeastern Brazil evidenced increased risk of oral cancer in patients presenting smoking and alcohol consumption synergism compared to non-synergetic consumption of cigarettes and alcohol (Andrade et al., 2015; Dias et al., 2017).

Regarding, the GST gene family polymorphisms, the GSTT1 and GSTM1 null genotype was not associated with the HNSCC carcinogenesis. Several studies have evaluated the association between these polymorphisms and head and neck cancer, however, the results are inconsistent (Kweon et al., 2014; He et al., 2016; Singh and Ghosh, 2019). A recent meta-analysis evaluated the effects of GSTT1, GSTM1 null genotypes on the risk of oral cancer in the Chinese population, this study included 1,306 oral cancer cases and 1,484 controls and did not find a significant association for the two evaluated polymorphisms. In another study, the stratified analysis by ethnicity demonstrated significant risks for GSTM1 null genotypes and head and neck cancer among Asians $(\mathrm{OR}=1.39,95 \% \mathrm{CI}=1.27-1.53 ; \mathrm{P}=0.000)$, but not in Caucasians $(\mathrm{OR}=0.99,95 \% \mathrm{CI}=0.83-1.18 ; \mathrm{P}=0.677)$, thus corroborating our results (Li et al., 2018).

The GSTT1 null genotype was less frequent among the investigated patients and healthy individuals and may indicate a reduced risk for cancer development. Similarly, studies on patients with HNSCC, such as the study carried in Brazilian population observed a lower frequency of the GSTT1 null genotype (16.8\%) in healthy subjects (Brunialti, 2009), while Silva et al. found a higher frequency of the GSTT1 positive genotype among healthy individuals (24.1\%) (Silva et al., 2014).

GSTT1 deletion leads to decreased ability to detoxify carcinogens efficiently and thus may increase ESCC risk. It has been consistently observed that those with homozygous GSTT1 null genotypes are at a higher risk of cancer, which supports the involvement of GSTT1 polymorphisms in ESCC carcinogenesis (Makhdoomi et al., 2015). As the effect of any single gene probably has a limited impact on cancer risk, the combination of certain genotypes may be more effective. In this study, no association was found for this genotype alone, but we were able to demonstrate that the GSTT1null + GSTM1 positive association increased the risk for HNSCC. A recent study from our research group demonstrated a protective association of GSTT1 null genotype and colorectal cancer $(\mathrm{OR}=0.65,95 \% \mathrm{CI}=0.43-0.98, \mathrm{P}=0.037)$, which may justify our meeting for this genotype alone (Rodrigues-Fleming et al., 2018).

Considering GSTP1 polymorphisms, no association was possible between the GSTP1-C341T and the risk for 
HNSCC. However, this association was confirmed for the GSTP1-313 A/G + G/G genotypes polymorphism, where at least one polymorphic allele present confers reduced risk for head and neck cancer. The study carried out by Russo et al. (Russo et al., 2013), also in a Brazilian population with HNSCC, indicated that A313G GSTP1 $\mathrm{AG} / \mathrm{GG}$ genotypes were associated with reduced risk for developing HNSCC, agreeing with the associations observed in the present study for this polymorphism.

Genotypic frequencies for the $\mathrm{A} 313 \mathrm{G}$ and $\mathrm{C} 341 \mathrm{~T}$ GSTP1 polymorphisms were in Hardy-Weinberg equilibrium in both groups ( $\mathrm{p}>0.05)$. The A313G/C341T GSTP1 haplotypes (Table 3) revealed a higher frequency of the two wild alleles (A-C) in the case group than in the control group (72.0 and 65.0, respectively; $\mathrm{p}=0.013$ ). Similarly, a higher frequency of the A-T haplotype was also found in the case group than in the control group (3.0 and 0.9 , respectively; $p=0.019$ ). However, the opposite was observed for the G-C haplotype, which was more frequent in the control group than in the case group (23.0 and 30.0, respectively; $\mathrm{p}=0.015$ ).

The present study failed to statistically establish these associations and indicates that studies with larger sample numbers may better demonstrate the relation between the response to the chemotherapy and GSTs polymorphisms (Ruwali et al., 2009). On the other hand, the Indian population has demonstrated an association between null GSTM1 genotypes and toxicity observed due to cisplatin (Dhawan et al., 2013).

Our findings did not indicate a relationship between overall survival and the studied genotypes. The Brazilian study corroborates the findings related to survival and the GSTP1 gene, but that study investigated the expression of this gene (Soares et al., 2017). Besides, reports of a trend towards significance $(\mathrm{p}=0.053)$ between the GSTT1 deletion polymorphism and progression-free survival in gastroesophageal adenocarcinoma are also found in the literature (Goekkurt et al., 2009).

The association of radiotherapy and/or chemotherapy in HNSCC treatment is evidenced by the literature and is indicated when it is impossible to perform surgery at the cancer site, as in the case of advanced tumors that affect important regions. Also, advanced tumors are more frequent when observing outpatient routine (Ruwali et al., 2009), as well as the findings obtained in the present study. Studies report that late HNSCC diagnosis can occur due to the absence of symptoms in most initial lesions, as well as due to lack of knowledge concerning differential diagnoses by some health professionals, contributing to late diagnosis with advanced-stage tumors (T3 and T4), already requiring radiotherapy and/or chemotherapy (Machiels et al., 2014).

In the present study, it was not possible to establish associations between primary anatomical site, clinical-pathological tumor features, relapse-free time and survival of HNSCC patients. To the primary anatomic site, a previous study in Brazilian HNSCC patients found no association of the GSTT1 null genotype with increased risk for larynx and decreased risk for pharynx cancers, thus, indicating that this polymorphism may modulate the risk to develop HNSCC at this anatomical site. The same study also found no association between the investigated GST polymorphisms with other clinical-pathological tumor features (Russo et al., 2013).

The predisposition to head and neck cancer is multifactorial and results from the interaction between allelic variant genes and environmental factors, such as old age, eating habits, and smoking and drinking habits. Therefore, the findings regarding the modulation of cancer susceptibility in the presence of the analyzed polymorphisms reinforce the influence on the etiology of the disease, although they do not influence patient survival. These results may contribute to the understanding of the mechanisms involved in the carcinogenesis of head and neck cancer. In conclusion, the present study indicates that age $\geq 59$, male sex, smoking and drinking habits are risk factors for HNSCC development. The presence of the GSTM1 null genotype is associated with increased risk for HNSCC, while the GSTT1 null genotype and the $A 313 G$ GSTP1 polymorphism contribute to decreased risk for this tumor type. The GSTM1, GSTT1 and GSTP1 gene polymorphisms are not associated with tumor site, tumor extension, regional lymph node involvement, and tumor progression. Polymorphisms are also not associated with relapse-free time or survival time of patients treated with chemotherapy and/or radiotherapy.

\section{Ethics statement}

All the procedures performed in this study involving human participants were in accordance with the norms in force of the Ethics Committee on Human Research of our institution in Brazil CEP (Committee of Ethics and Research) / CONEP (National Commission of Ethics in Research) and São José do Rio Preto School of Medicine, (FAMERP), No. 697.896 and patients gave their informed consent before the material was obtained for use in the study. This article does not contain animal studies by any of the authors.

\section{Acknowledgments}

The authors are grateful to the participating patients, their families and to the team of the Biological and Genetics and Molecular Biology Research Unit - UPGEM that in some way supported the development of this study. The authors also thank the financial support of the Foundation for Research Support of the State of São Paulo - FAPESP (2007/08155-2), the Coordenação de Aperfeiçoamento de Pessoal de Nível Superior - CAPES (Financial Code 001) and Conselho Nacional de Desenvolvimento Científico e Tecnológico - CNPq (Processo n ${ }^{\circ}$ 310987/2018-0), and FAMERP/ FUNFARME support.

\section{Informed consent}

Informed consent was obtained from all individual participants included in the study.

\section{Conflict of interest}

The authors declare that they have no conflict of interest.

\section{Author contributions}

Asian Pacific Journal of Cancer Prevention, Vol 211643 
Mauricio Pereira Maniglia planned and conducted the study, collected and interpreted data, and drafted and wrote the manuscript; Anelise Russo participated in the collection of the genetic material, interpreted data, and drafted and wrote the manuscript; Gabriela Helena Rodrigues-Fleming and Juliana Garcia de Oliveira-Cucolo participated analysis and interpreted data, and revised the manuscript; Patricia Matos Biselli-Chicote critically revised the statistical and the manuscript; José Victor Maniglia provided the patients for the research and served as a scientific advisor; Érika Cristina Pavarino served as a scientific advisor; Eny Maria Goloni- Bertollo was the guarantor, planned the study, and critically revised the manuscript.

\section{References}

Abdel-Rahman SZ, el-Zein RA, Anwar WA, et al (1996). A multiplex PCR procedure for polymorphic analysis of GSTM1 and GSTT1 genes in population studies. Cancer Lett, 107, 229-33.

Andrade JO, Santos CA, Oliveira MC (2015). Associated factors with oral cancer: a study of case control in a population of the Brazil's Northeast. Rev Bras Epidemiol, 18, 894-905.

Carron J, Lopes-Aguiar L, Costa EFD, et al (2017). GSTP1 c. $313 \mathrm{~A}>\mathrm{G}$, XPD c. $934 \mathrm{G}>\mathrm{A}, \mathrm{XPF}$ c. $2505 \mathrm{~T}>\mathrm{C}$ and $\mathrm{CASP} 9$ c. $-1339 A>G$ polymorphisms and severity of vomiting in head and neck cancer patients treated with cisplatin chemoradiation. Basic Clin Pharmacol Toxicol, 121, 520-5.

Chatterjee A, Gupta S (2018). The multifaceted role of glutathione S-transferases in cancer. Cancer Lett, 433, 33-42.

Choudhury JH, Singh SA, Kundu S, et al (2015). Tobacco carcinogen-metabolizing genes CYP1A1, GSTM1, and GSTT1 polymorphisms and their interaction with tobacco exposure influence the risk of head and neck cancer in Northeast Indian population. Tumour Biol, 36, 5773-83.

Dhawan D, Panchal H, Shukla S, et al (2013). Genetic variability \& chemotoxicity of 5-fluorouracil \& cisplatin in head \& neck cancer patients: a preliminary study. Indian J Med Res, 137, 125-9.

Edge SB, Compton CC (2010). The American Joint Committee on Cancer: the 7th edition of the AJCC cancer staging manual and the future of TNM. Ann Surg Oncol, 17, 1471-4.

Galbiatti AL, Padovani-Junior JA, Maníglia JV, et al (2013). Head and neck cancer: causes, prevention and treatment. Braz J Otorhinolaryngol, 79, 239-47.

Goekkurt E, Al-Batran SE, Hartmann JT, et al (2009). Pharmacogenetic analyses of a phase III trial in metastatic gastroesophageal adenocarcinoma with fluorouracil and leucovorin plus either oxaliplatin or cisplatin: a study of the arbeitsgemeinschaft internistische onkologie. J Clin Oncol, 27, 2863-73.

Harries LW, Stubbins MJ, Forman D, et al (1997). Identification of genetic polymorphisms at the glutathione S-transferase Pi locus and association with susceptibility to bladder, testicular and prostate cancer. Carcinogenesis, 18, 641-4.

Hasegawa Y, Goto M, Hanai N, et al (2018). Predictive biomarkers for combined chemotherapy with 5-fluorouracil and cisplatin in oro- and hypopharyngeal cancers. Mol Clin Oncol, 8, 378-86.

He P, Wei M, Wang Y, et al (2016). Associations among Glutathione S-Transferase T1, M1, and P1 polymorphisms and the risk of oral leukoplakia. Genet Test Mol Biomarkers, 20, 312-21.

Kweon SS, Shin MH, Kim HN, et al (2014). Polymorphisms of methylenetetrahydrofolate reductase and glutathione S-transferase are not associated with the risk of papillary thyroid cancer in Korean population. Mol Biol Rep, 41, 3793-9.

Li JY, Huang LN, Xue HL, et al (2018). Glutathione S-transferase mu-1, glutathione S-transferase theta-1 null genotypes, and oral cancer risk: A meta-analysis in the Chinese population. $J$ Cancer Res Ther, 14, 1052-6.

Macedo LT, Ferrari VE, Carron J, et al (2019). Cost-minimization analysis of GSTP1c.313A $>$ G genotyping for the prevention of cisplatin-induced nausea and vomiting: A Bayesian inference approach. PLoS One, 14, e0213929.

Machiels JP, Lambrecht M, Hanin FX, et al (2014). Advances in the management of squamous cell carcinoma of the head and neck. F1000Prime Rep, 6, 44.

Makhdoomi MA, Shah IA, Bhat GA, et al (2015). Association between GSTM1 and GSTT1 polymorphisms and esophageal squamous cell carcinoma: results from a case-control study in Kashmir, India. Tumour Biol, 36, 2613-9.

Marchewka Z, Piwowar A, Ruzik S, et al (2017). Glutathione $\mathrm{S}$ - transferases class $\mathrm{Pi}$ and $\mathrm{Mi}$ and their significance in oncology. Postepy Hig Med Dosw (Online), 71, 541-50.

Mirghani H, Sturgis EM, Aupérin A, et al (2017). Is there an increased risk of cancer among spouses of patients with an HPV-related cancer: A systematic review. Oral Oncol, 67, 138-45.

Rodrigues-Fleming GH, Fernandes GMM, Russo A, et al (2018). Molecular evaluation of glutathione $\mathrm{S}$ transferase family genes in patients with sporadic colorectal cancer. World $J$ Gastroenterol, 24, 4462-71.

Russo A, Francelin PR, Galbiatti AL, et al (2013). Association between GSTP1, GSTM1 and GSTT1 polymorphisms involved in xenobiotic metabolism and head and neck cancer development. Mol Biol Rep, 40, 4181-8.

Ruwali M, Pant MC, Shah PP, et al (2009). Polymorphism in cytochrome P450 2A6 and glutathione S-transferase P1 modifies head and neck cancer risk and treatment outcome. Mutat Res, 669, 36-41.

Salazar LA, Hirata MH, Cavalli SA, et al (1998). Optimized procedure for DNA isolation from fresh and cryopreserved clotted human blood useful in clinical molecular testing. Clin Chem, 44, 1748-50.

Silva TM, Marques CR, Marques Filho MF, et al (2014). Association of the GSTT1 polymorphism in upper aerodigestive tract cancer with tobacco smoking. Genet Mol Res, 13, 528-37.

Singh SA, Ghosh SK (2019). Metabolic phase I (CYPs) and phase II (GSTs) gene polymorphisms and their interaction with environmental factors in nasopharyngeal cancer from the ethnic population of northeast India. Pathol Oncol Res, 25, 33-44.

Soares PO, Maluf Cury P, Mendoza López RV, et al (2017). GTSP1 expression in non-smoker and non-drinker patients with squamous cell carcinoma of the head and neck. PLoS One, 12, e0182600.

Watson MA, Stewart RK, Smith GB, et al (1998). Human glutathione S-transferase P1 polymorphisms: relationship to lung tissue enzyme activity and population frequency distribution. Carcinogenesis, 19, 275-80.

Zhang Y, Zhang H, Lin P, et al (2019). Glutathione S-transferase gene polymorphisms and risk of nasal or colorectal polyposis. Biosci Rep, 39.

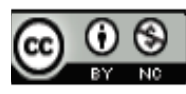

This work is licensed under a Creative Commons AttributionNon Commercial 4.0 International License. 